\title{
Unravelling Ion Dynamics and Interactions in an Ionic Liquid Electrolyte with a Protonated Anion for Lithium Batteries
}

Derick Gyabeng a ${ }^{a}$ Lixin Qiao ${ }^{\text {b,c }}$, Heng Zhang ${ }^{d}$, Uxue Oteo ${ }^{b}$, Michel Armand ${ }^{\text {b }}$, Maria Forsyth $^{\text {a }}$, Fangfang Chen ${ }^{* a, e}$, and Luke A. O’Dell *a

a Institute for Frontier Materials, Deakin University, Geelong VIC 3220, Australia

${ }^{b}$ Centre for Cooperative Research on Alternative Energies (CIC energiGUNE), Basque Research and Technology Alliance (BRTA), Alava Technology Park, Albert Einstein 48, 01510 Vitoria-Gasteiz, Spain

${ }^{c}$ University of the Basque Country (UPV/EHU), Barrio Sarriena, s/n, 48940 Leioa, Spain ${ }^{d}$ Key Laboratory of Material Chemistry for Energy Conversion and Storage (Ministry of Education), School of Chemistry and Chemical Engineering, Huazhong University of Science and Technology, Luoyu Road 1037, 430074, Wuhan, China

e ARC Centre of Excellence for Electromaterials Science (ACES), Deakin University, Burwood, 3125, Australia 


\section{Density of the ILs}

Table S1. The specific density $(\rho)$ from 3 repetitions and the corresponding average density ( $\bar{\rho})$, standard deviation $\left(\rho_{\mathrm{SD}}\right)$ and functional uncertainty $\left(f_{\mathrm{FN}}\right)$ for the ILs.

\begin{tabular}{lcccccc}
\hline & $\begin{array}{l}\rho_{1} / \mathrm{kg} \\
\mathrm{L}^{-1}\end{array}$ & $\begin{array}{l}\rho_{2} / \mathrm{kg} \\
\mathrm{L}^{-1}\end{array}$ & $\begin{array}{l}\rho_{3} / \mathrm{kg} \\
\mathrm{L}^{-1}\end{array}$ & $\bar{\rho} / \mathrm{kg} \mathrm{L}^{-1}$ & $\rho_{\mathrm{SD}} / \mathrm{kg} \mathrm{L}^{-1}$ & $f_{\mathrm{FN}} / \%$ \\
\hline $\begin{array}{l}\mathrm{C}_{3} \text { mpyr } \\
\text { LiDFTFSI }\end{array}$ & 1.47 & 1.46 & 1.44 & 1.46 & 0.014 & 0.6 \\
$\begin{array}{l}\mathrm{C}_{2 \mathrm{O} 1 \text { mpyr }} \\
\text { LiDFTFSI }\end{array}$ & 1.43 & 1.61 & 1.57 & 1.54 & 0.097 & 3.6
\end{tabular}

The experimental repetition uncertainties were systematically studied for density of the ILs, The standard deviation $\left(\rho_{\mathrm{SD}}\right)$ and fractional uncertainty $\left(f_{\mathrm{FN}}\right)$ of density were calculated using Eq. S [1], Eq. S [2], ${ }^{1}$ respectively:

$$
\begin{array}{cc}
\rho_{\mathrm{SD}}=\sqrt{\sum\left(\rho_{\mathrm{i}}-\bar{\rho}\right)^{2} /(N-1)} & \mathrm{S}[1] \\
f_{\mathrm{FN}}=\rho_{\mathrm{SD}} /(\sqrt{N} \times \bar{\rho}) \times 100 \% & \mathrm{~S}[2]
\end{array}
$$

In Eq. S [1] and Eq. S [2], $\rho_{\mathrm{i}}$ is the density of sample $i, \bar{\rho}$ is the mean value of all the measured samples, and $N$ is the number of measurements. The calculated values are shown in Table S1. 


\section{NMR spectra of LIDFTFSI}

${ }^{1} \mathrm{H}$ NMR

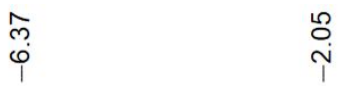
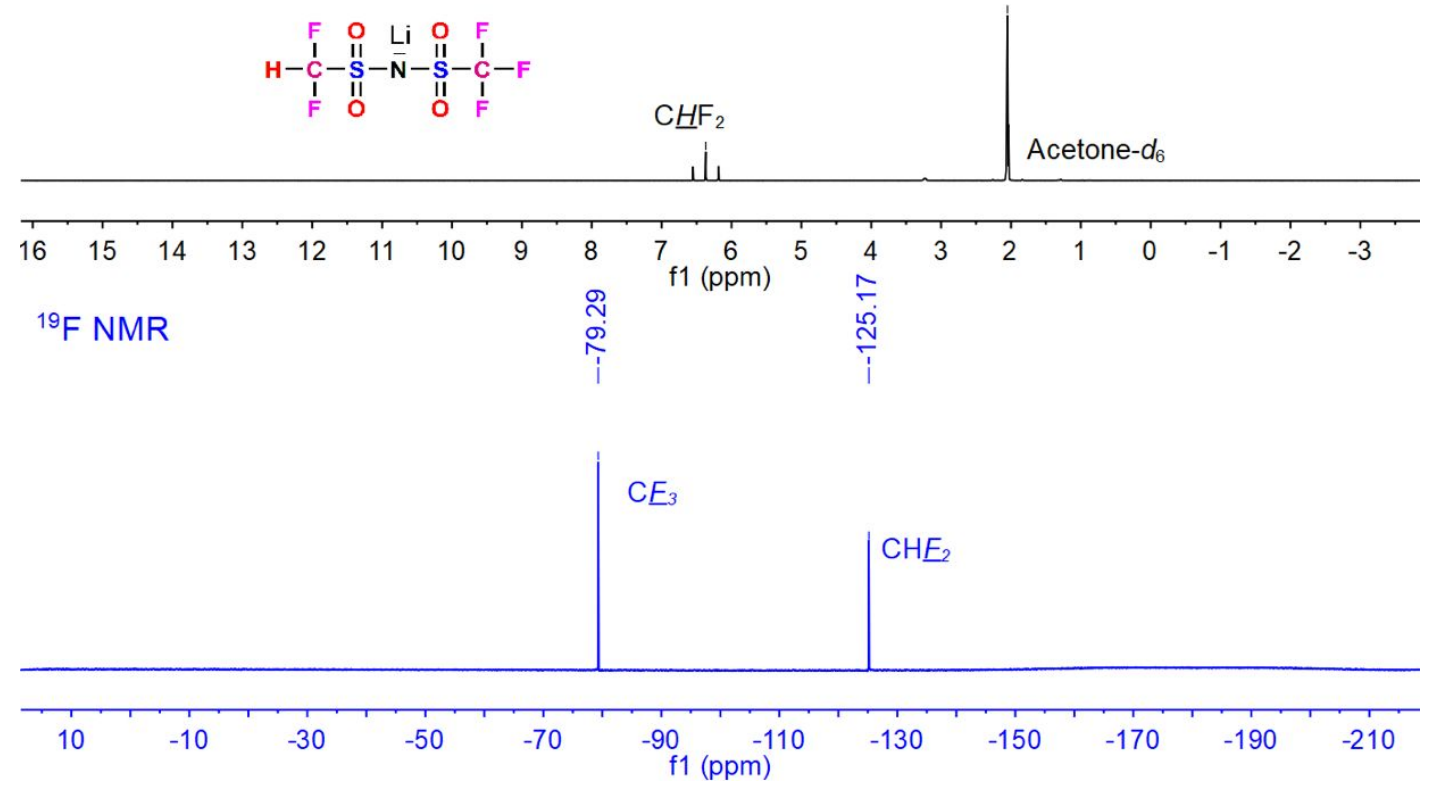

Figure S1: NMR spectra of the LiDFTFSI salt. The deuterated solvent for the NMR measurement is acetone- $d_{6}$. 


\section{Temperature dependent correlation times of different nuclei in $\mathrm{C}_{3} \mathrm{mpyr}$ LiDFTFSI}

Table S2: Correlation times from ${ }^{19} \mathrm{~F},{ }^{1} \mathrm{H}$ and ${ }^{7} \mathrm{Li}$ nuclei $\mathrm{T}_{1}$ relaxation measurements extracted from BPP fitting at varying temperatures in $\mathrm{C}_{3}$ mpyr LiDFTFSI.

\begin{tabular}{|c|c|c|c|c|c|c|}
\hline & \multicolumn{2}{|c|}{${ }^{19} \mathbf{F}$} & \multicolumn{3}{c|}{${ }^{1} \mathbf{H}$} & ${ }^{7} \mathbf{L}$ \\
\hline $\begin{array}{c}\text { Temperature } \\
(\mathbf{K})\end{array}$ & $\mathbf{C F}_{3}$ & $\mathbf{C F}_{2} \mathbf{H}$ & Anion & $\mathbf{C 2}$ & $\mathbf{C 6}$ & \\
\hline 253 & $6.41 \mathrm{E}-10$ & $9.78 \mathrm{E}-10$ & $9.52 \mathrm{E}-10$ & $7.91 \mathrm{E}-10$ & $4.34 \mathrm{E}-10$ & $1.50 \mathrm{E}-08$ \\
\hline 263 & $4.99 \mathrm{E}-10$ & $7.55 \mathrm{E}-10$ & $6.89 \mathrm{E}-10$ & $5.86 \mathrm{E}-10$ & $3.53 \mathrm{E}-10$ & $1.06 \mathrm{E}-08$ \\
\hline 273 & $3.96 \mathrm{E}-10$ & $5.95 \mathrm{E}-10$ & $5.11 \mathrm{E}-10$ & $4.44 \mathrm{E}-10$ & $2.92 \mathrm{E}-10$ & $7.71 \mathrm{E}-09$ \\
\hline 283 & $3.19 \mathrm{E}-10$ & $4.76 \mathrm{E}-10$ & $3.86 \mathrm{E}-10$ & $3.43 \mathrm{E}-10$ & $2.44 \mathrm{E}-10$ & $5.72 \mathrm{E}-09$ \\
\hline 293 & $2.61 \mathrm{E}-10$ & $3.87 \mathrm{E}-10$ & $2.98 \mathrm{E}-10$ & $2.70 \mathrm{E}-10$ & $2.07 \mathrm{E}-10$ & $4.33 \mathrm{E}-09$ \\
\hline 303 & $2.17 \mathrm{E}-10$ & $3.19 \mathrm{E}-10$ & $2.34 \mathrm{E}-10$ & $2.16 \mathrm{E}-10$ & $1.78 \mathrm{E}-10$ & $3.34 \mathrm{E}-09$ \\
\hline 313 & $1.82 \mathrm{E}-10$ & $2.66 \mathrm{E}-10$ & $1.86 \mathrm{E}-10$ & $1.75 \mathrm{E}-10$ & $1.54 \mathrm{E}-10$ & $2.62 \mathrm{E}-09$ \\
\hline 323 & $1.54 \mathrm{E}-10$ & $2.24 \mathrm{E}-10$ & $1.51 \mathrm{E}-10$ & $1.44 \mathrm{E}-10$ & $1.34 \mathrm{E}-10$ & $2.08 \mathrm{E}-09$ \\
\hline 333 & $1.32 \mathrm{E}-10$ & $1.91 \mathrm{E}-10$ & $1.23 \mathrm{E}-10$ & $1.19 \mathrm{E}-10$ & $1.18 \mathrm{E}-10$ & $1.68 \mathrm{E}-09$ \\
\hline 343 & $1.14 \mathrm{E}-10$ & $1.64 \mathrm{E}-10$ & $1.02 \mathrm{E}-10$ & $1.00 \mathrm{E}-10$ & $1.05 \mathrm{E}-10$ & $1.37 \mathrm{E}-09$ \\
\hline 353 & $9.97 \mathrm{E}-11$ & $1.43 \mathrm{E}-10$ & $8.55 \mathrm{E}-11$ & $8.50 \mathrm{E}-11$ & $9.35 \mathrm{E}-11$ & $1.14 \mathrm{E}-09$ \\
\hline & & & & & & \\
\hline
\end{tabular}




\section{Temperature dependent correlation times of different nuclei in $\mathrm{C}_{201}$ mpyr LiDFTFSI}

Table S3: Correlation times from ${ }^{19} \mathrm{~F},{ }^{1} \mathrm{H}$ and ${ }^{7} \mathrm{Li}$ nuclei $\mathrm{T}_{1}$ relaxation measurements extracted from BPP fitting at varying temperatures in $\mathrm{C}_{3}$ mpyr LiDFTFSI.

\begin{tabular}{|c|c|c|c|c|c|c|}
\hline & \multicolumn{2}{|c|}{${ }^{19} \mathbf{F}$} & \multicolumn{3}{c|}{${ }^{1} \mathbf{H}$} & ${ }^{7} \mathbf{L}$ \\
\hline $\begin{array}{c}\text { Temperature } \\
(\mathbf{K})\end{array}$ & $\mathbf{C F}_{3}$ & $\mathbf{C F}_{2} \mathbf{H}$ & Anion & $\mathbf{C} 2$ & $\mathbf{C 6}$ & \\
\hline 253 & $5.97 \mathrm{E}-10$ & $1.04 \mathrm{E}-09$ & $1.03 \mathrm{E}-09$ & $1.07 \mathrm{E}-09$ & $5.69 \mathrm{E}-10$ & $1.15 \mathrm{E}-08$ \\
\hline 263 & $4.64 \mathrm{E}-10$ & $7.88 \mathrm{E}-10$ & $7.28 \mathrm{E}-10$ & $7.61 \mathrm{E}-10$ & $4.43 \mathrm{E}-10$ & $8.12 \mathrm{E}-09$ \\
\hline 273 & $3.67 \mathrm{E}-10$ & $6.09 \mathrm{E}-10$ & $5.27 \mathrm{E}-10$ & $5.57 \mathrm{E}-10$ & $3.51 \mathrm{E}-10$ & $5.88 \mathrm{E}-09$ \\
\hline 283 & $2.96 \mathrm{E}-10$ & $4.79 \mathrm{E}-10$ & $3.90 \mathrm{E}-10$ & $4.17 \mathrm{E}-10$ & $2.82 \mathrm{E}-10$ & $4.36 \mathrm{E}-09$ \\
\hline 293 & $2.41 \mathrm{E}-10$ & $3.83 \mathrm{E}-10$ & $2.95 \mathrm{E}-10$ & $3.18 \mathrm{E}-10$ & $2.31 \mathrm{E}-10$ & $3.30 \mathrm{E}-09$ \\
\hline 303 & $2.00 \mathrm{E}-10$ & $3.11 \mathrm{E}-10$ & $2.27 \mathrm{E}-10$ & $2.47 \mathrm{E}-10$ & $1.91 \mathrm{E}-10$ & $2.54 \mathrm{E}-09$ \\
\hline 313 & $1.67 \mathrm{E}-10$ & $2.55 \mathrm{E}-10$ & $1.78 \mathrm{E}-10$ & $1.95 \mathrm{E}-10$ & $1.60 \mathrm{E}-10$ & $1.99 \mathrm{E}-09$ \\
\hline 323 & $1.42 \mathrm{E}-10$ & $2.13 \mathrm{E}-10$ & $1.41 \mathrm{E}-10$ & $1.57 \mathrm{E}-10$ & $1.36 \mathrm{E}-10$ & $1.58 \mathrm{E}-09$ \\
\hline 333 & $1.21 \mathrm{E}-10$ & $1.79 \mathrm{E}-10$ & $1.14 \mathrm{E}-10$ & $1.27 \mathrm{E}-10$ & $1.16 \mathrm{E}-10$ & $1.27 \mathrm{E}-09$ \\
\hline 343 & $1.05 \mathrm{E}-10$ & $1.52 \mathrm{E}-10$ & $9.28 \mathrm{E}-11$ & $1.05 \mathrm{E}-10$ & $1.00 \mathrm{E}-10$ & $1.04 \mathrm{E}-09$ \\
\hline 353 & $9.12 \mathrm{E}-11$ & $1.30 \mathrm{E}-10$ & $7.66 \mathrm{E}-11$ & $8.69 \mathrm{E}-11$ & $8.75 \mathrm{E}-11$ & $8.59 \mathrm{E}-10$ \\
\hline
\end{tabular}

\section{Reference}

(1) Cohen, E. R. An Introduction to Error Analysis: The Study of Uncertainties in Physical Measurements. Measurement Science and Technology 1998, 9 (6), 191-192. 